\title{
MAGNETIC GAMES BETWEEN A PLANET AND ITS HOST STAR: THE KEY ROLE OF TOPOLOGY
}

\author{
A. StrugareK ${ }^{1,2}$, A. S. Brun ${ }^{2}$, S. P. MatT ${ }^{3}$, and V. Réville ${ }^{2}$ \\ ${ }^{1}$ Département de physique, Université de Montréal, C.P. 6128 Succ. Centre-Ville, Montréal, QC H3C 3J7, Canada; strugarek@astro.umontreal.ca \\ ${ }^{2}$ Laboratoire AIM Paris-Saclay, CEA/Irfu Université Paris-Diderot CNRS/INSU, F-91191 Gif-sur-Yvette, France \\ ${ }^{3}$ Astrophysics group, School of Physics, University of Exeter, Stocker Road, Exeter EX4 4QL, UK \\ Received 2015 October 2; accepted 2015 November 9; published 2015 December 15
}

\begin{abstract}
Magnetic interactions between a star and a close-in planet are postulated to be a source of enhanced emissions and to play a role in the secular evolution of the orbital system. Close-in planets generally orbit in the sub-alfvénic region of the stellar wind, which leads to efficient transfers of energy and angular momentum between the star and the planet. We model the magnetic interactions occurring in close-in star-planet systems with three-dimensional, global, compressible magnetohydrodynamic numerical simulations of a planet orbiting in a self-consistent stellar wind. We focus on the cases of magnetized planets and explore three representative magnetic configurations. The Poynting flux originating from the magnetic interactions is an energy source for enhanced emissions in star-planet systems. Our results suggest a simple geometrical explanation for ubiquitous on/off enhanced emissions associated with close-in planets, and confirm that the Poynting fluxes can reach powers of the order of $10^{19} \mathrm{~W}$. Close-in planets are also shown to migrate due to magnetic torques for sufficiently strong stellar wind magnetic fields. The topology of the interaction significantly modifies the shape of the magnetic obstacle that leads to magnetic torques. As a consequence, the torques can vary by at least an order of magnitude as the magnetic topology of the interaction varies.
\end{abstract}

Key words: magnetohydrodynamics (MHD) - planet-star interactions - planets and satellites: dynamical evolution and stability - stars: winds, outflows

\section{INTRODUCTION}

Techniques for the detection of exoplanets favor so far the discovery of giant, close-in planets that can significantly perturb radial velocity signals, or lead to deep, well defined transits. Furthermore, $34 \%$ of the known exoplanets ${ }^{4}$ are in orbit closer than $20 R_{\star}$. Such planets are expected to interact strongly with their hosts (see, e.g., Cuntz et al. 2000) in a potentially observable way. The interactions originate from tides, magnetism, and radiative processes. The proximity of close-in exoplanets amplifies these effects, which can theoretically lead to exchanges of energy and angular momentum between the star and the planet and may have observable signatures.

As a matter of fact, several intriguing observations are associated with close-in exoplanets. Shkolnik et al. (2008, and references therein) reported chromospheric emissions for five different star-planet pairs that correlate with the planetary orbital period. These correlated emissions were observed to be subject to an on/off mechanism, possibly originating from the variability of the stellar magnetic field over timescales of years, or over the orbital phase of the planet. The particular case of HD 189733 was recently revisited by Pillitteri et al. (2015), who interpreted the excess emission as resulting from an infall of planetary material toward the star. The surprising lack of $\mathrm{X}$-ray emissions from WASP-18 is also thought to result from some star-planet interaction (Pillitteri et al. 2014), which is yet to be understood. Nevertheless, it is today clear that the enhancement (or lack thereof) of chromospheric or coronal $\mathrm{X}$-ray emissions due to a close-in planet is situational and highly variable: it does not statistically induce an observational trend (see Miller et al. 2015, and references therein). Radio and UV emissions from star-planet magnetic interactions (SPMI)

\footnotetext{
4 From the database exoplanet.eu, 2015 September 1.
}

are also intensively researched today (Grießmeier et al. 2007; Fares et al. 2010; Lecavelier des Etangs et al. 2013; Turner et al. 2013), because any detection may provide constraints on the hypothetical planetary magnetic fields (e.g., Zarka 2007; Vidotto et al. 2015).

The statistical distribution of exoplanets also reveals interesting features. First noted by Pont (2009), it appears that hosts of close-in planets tend to rotate more rapidly than twin stars hosting no close-in planets. This was recently confirmed by Maxted et al. (2015), although the authors question the original explanation of Pont (2009) based on tidal interactions because they do not seem to find a correlation between the anomalous gyrochronological age and the strength of the expected tidal forces. Furthermore, McQuillan et al. (2013) and Lanza \& Shkolnik (2014) showed a clear dearth of close-in exoplanets around fast rotators with Kepler. Both effects could be explained by exchanges of angular momentum between stars and close-in planets, although their detailed mechanism is still debated today.

The aforementioned observations are generally interpreted in terms of tidal, radiative, or magnetic star-planet interactions. Tides are known to lead to spin-orbit synchronization in starplanet systems (for a review, see Mathis et al. 2013). The angular momentum transport resulting from star-planet tides can also lead to planet migration (see, e.g., Bolmont et al. 2012; Zhang \& Penev 2014; Damiani \& Lanza 2015) as well as spinup the host star for close-in planets (Barker \& Ogilvie 2011; Poppenhaeger \& Wolk 2014; Ferraz-Mello et al. 2015). Planetdisk tidal interactions provide as well various migration mechanisms (for a review, see Baruteau et al. 2014) in the early stages of stellar systems. The efficiency of tidal interactions depends strongly on the internal structure of both the star and the planet, and its modeling is still a subject of 
intense research today (Auclair-Desrotour et al. 2014; Guenel et al. 2014).

Close-in planets are also subject to intense radiation from their host that can lead to planetary outflows (see, e.g., Yelle et al. 2008; Owen \& Adams 2014; Trammell et al. 2014) and may in some cases allow some planetary material to impact the stellar chromosphere. In the case of close-in unmagnetized planets, intense extreme-UV radiation was shown to favor the penetration of the stellar wind and may be a source of enhanced atmospheric escape (Cohen et al. 2015). Matsakos et al. (2015) classified the different types of planetary outflows, though further investigation is still required to elucidate how such flows could explain enhanced emissions or statistical trends in the exoplanet population (see Pillitteri et al. 2015, for a possible link between hot spots and radiation-induced planetary outflows).

Magnetic interactions provide another promising mechanism for the transfer of energy and angular momentum between a star and a planet. A close-in planet generally orbits inside the sub-alfvénic region of the stellar wind, leading to particularly efficient transfers (see, e.g., Cohen et al. 2010; Strugarek et al. 2014b). In a pioneering work Ip et al. (2004) modeled such interaction as a plausible source of additional and localized coronal heating of close-in planet-hosting stars. In this scenario, in contrast to radiatively induced planetary outflows, the energy is carried away from the planet by alfvénic perturbations propagating in the stellar wind down to the stellar chromosphere. The energetic transfers occurring due to magnetic interactions in a star-planet system can be modeled with the concept of Alfvén wings (Neubauer 1998), inspired by similar planet-satellite magnetic interactions occurring in the solar system (Goldreich \& Lynden-Bell 1969; Neubauer 1980). However, in star-planet systems, the detailed structure of the wind determines how the Alfvén wings develop. The Poynting flux in Alfvén wings was quantified by Saur et al. (2013) for the exoplanets known at that time, using simple 1D stellar wind models. This could provide a source for the intermittent enhanced emissions sometimes observed in close-in exosystems. The magnetic torques originating from SPMI were proposed as well to be a source of planet migration (Laine et al. 2008; Lovelace et al. 2008; Vidotto et al. 2010; Laine \& Lin 2011; Strugarek et al. 2014b) and stellar spin-up (Cohen et al. 2010; Lanza 2010; Strugarek et al. 2014b). On the contrary, for fast rotating stars those magnetic torques can spin-down the star, albeit not efficiently enough to solve the so-called angular momentum problem for young stars (Bouvier \& Cébron 2015). As in the case of tidal interactions, it is important to note that SPMI generally depend on the planet's internal composition, and in particular on whether or not a dynamo process is able to sustain an intrinsic magnetic field in its interior (Strugarek et al. 2014b).

This paper focuses on the effects of magnetic topology in the development of magnetic interactions between a star and a close-in, magnetized planet. We investigate whether magnetic interactions can be strong enough to explain enhanced emissions or a statistical dearth of close-in planets around fast rotating stars. We study how Alfvén wings develop in selfconsistent, global three-dimensional numerical models of stellar winds in which an orbiting, magnetized planet is added. We explore three extreme magnetic topologies of aligned, antialigned, and perpendicular configurations. We systematically characterize the energy and angular momentum exchanges that occur in each case and demonstrate the crucial influence of the magnetic topology. In Section 2 we describe the modeling approach we chose for the stellar wind and the magnetized planet. A detailed study of the Alfvén wings that selfconsistently develop in our numerical model is given in Section 3. The magnetic torques leading to planet migration are characterized in Section 4 and conclusions are given in Section 5.

\section{STELLAR WIND AND PLANET MODELS}

We use the PLUTO code (Mignone et al. 2007) to model star-planet magnetic interactions. We detail here the system of equations we solve, our modeling choices for the stellar wind and the planet, and the numerical methods we use.

\subsection{Magnetohydrodynamic Equations}

The PLUTO code solves the following set of compressible, ideal magnetohydrodynamic (MHD) equations:

$$
\begin{gathered}
\partial_{t} \rho+\boldsymbol{\nabla} \cdot(\rho \boldsymbol{v})=0 \\
\rho \partial_{t} \boldsymbol{v}+\rho \boldsymbol{v} \cdot \boldsymbol{\nabla} \boldsymbol{v} \boldsymbol{\nabla}+\boldsymbol{B} \times \boldsymbol{\nabla} \times \boldsymbol{B} /(4 \pi)=\rho \boldsymbol{a}, \\
\partial_{t} P+\boldsymbol{v} \cdot \boldsymbol{\nabla} P+\rho c_{s}^{2} \boldsymbol{\nabla} \cdot \boldsymbol{v}=0, \\
\partial_{t} \boldsymbol{B}-\boldsymbol{\nabla} \times(\boldsymbol{v} \times \boldsymbol{B})=0, \\
\boldsymbol{\nabla} \cdot \boldsymbol{B}=0,
\end{gathered}
$$

where $\rho$ is the plasma density, $v$ its velocity, $P$ the gas pressure, $\boldsymbol{B}$ the magnetic field, $\boldsymbol{a}$ is composed of the gravity, Coriolis, and centrifugal forces (the MHD equations are written in a rotating reference frame that is specified in Section 2.3), and $c_{s}=\sqrt{\gamma P / \rho}$ is the sound speed $(\gamma$ is the adiabatic exponent, taken to be the equal to the ratio of specific heats). We use an ideal-gas equation of state

$$
\rho \varepsilon=P /(\gamma-1),
$$

where $\varepsilon$ is the internal energy per unit mass.

\subsection{Stellar Wind Models}

The base of our MHD modeling approach for stellar wind was originally developed by Washimi \& Shibata (1993), and extended later on by Keppens \& Goedbloed (1999), Matt \& Balick (2004), Matt \& Pudritz (2008), Matt et al. (2012), Strugarek et al. (2014b), and Réville et al. (2015a). In this work we further develop this approach by considering threedimensional stellar winds. We briefly explain here our modeling methodology and refer the interested reader to the aforementioned works for further details.

We model stellar winds using the MHD approximation to describe the stellar corona plasma. In order to simplify the model, we do not describe the heating mechanism of the corona itself (see Suzuki \& Inutsuka 2006), and instead consider our stellar boundary condition to represent the base of the corona. We prescribe there a thermal pressure gradient that drives an outward accelerating flow, the stellar wind. To mimic the additional complex heating that occurs in the lower corona and participates in the physical acceleration of stellar winds, we choose an effective adiabatic exponent $\gamma$ close to isothermal (in this work $\gamma=1.05$ ). This choice is made to ensure that our model, when applied to the Sun, achieves velocities compatible with the slow component of the solar wind observations at 1 AU (Washimi \& Shibata 1993; Matt \& Balick 2004). 
Table 1

Properties of Stellar Winds

\begin{tabular}{lcc}
\hline \hline & Dipolar Wind & Quadrupolar Wind \\
\hline$B_{\star}($ equator $)(\mathrm{G})$ & 12.4 & 11 \\
$\dot{M}\left(10^{-14} M_{\odot} \mathrm{yr}^{-1}\right)$ & 2.04 & 2.11 \\
$\dot{J}\left(10^{-11} M_{\odot} R_{\odot}^{2} \mathrm{yr}^{-2}\right)$ & 56.0 & 2.55 \\
$\left\langle R_{\mathrm{A}}\right\rangle\left(R_{\star}\right)$ & 18.0 & 3.78 \\
\hline
\end{tabular}

The stellar wind is then determined by the interplay between the thermally accelerated flow, the large-scale magnetic structures, the rotation of the star, and the coronal density (see, e.g., Réville et al. 2015a, 2015b). This latter parameter can be conveniently used as an adimensionalization parameter for the MHD equations. The other parameters are described in terms of characteristic velocities normalized to the escape velocity $v_{\mathrm{esc}}=\sqrt{2 G M_{\star} / R_{\star}}$. The thermal driving of the wind is considered to be spherically symmetric and set by the sound speed $c_{S}=\sqrt{\gamma P / \rho}$. The rotation of the star is supposed to be solid and specified by the rotation speed $v_{\text {rot }}=\Omega_{\star} R_{\star}$. Finally, the stellar magnetic field is prescribed by a given idealized topology (dipole or quadrupole) and an equatorial Alfvén speed $v_{\mathrm{A}}=B_{\star} / \sqrt{4 \pi \rho_{\star}}$, where $B_{\star}$ is the stellar magnetic field on the equatorial plane. We consider in this work that the magnetic pole is aligned with the rotation axis.

We initialize our simulation domain with a spherically symmetric Parker wind (Parker 1958) to which we add a magnetic field of a given topology and a given normalized Alfvén speed $v_{\mathrm{A}} / v_{\mathrm{esc}}$. The star is modeled as an internal spherical boundary condition centered at the middle of the computational domain. The boundary condition consists of three spherical layers under the stellar "surface" in which the gradient of the Parker wind pressure, the rotation rate of the star, and its magnetic field are imposed (see Strugarek et al. 2014a, 2014b, for full details on these boundary conditions).

We study two different stellar winds using dipolar and quadrupolar magnetic fields to explore the effects of the magnetic topology on SPMI. Both winds are driven by a normalized sound speed $c_{s} / v_{\mathrm{esc}}=0.222$ corresponding to a coronal temperature of $10^{6} \mathrm{~K}$ for a solar-like star. They both rotate slowly with $v_{\text {rot }} / v_{\text {esc }}=3.03 \times 10^{-3}$. The dipolar case is characterized by a normalized Alfvén speed of $v_{\mathrm{A}} / v_{\mathrm{esc}}=1$, and in the quadrupolar case $v_{\mathrm{A}} / v_{\mathrm{esc}}=3$. This ensures that the total pressure at the planet's orbit (dominated by the magnetic pressure, see Section 2.3) is equivalent in both winds.

Because the coronal base density $\rho_{\star}$ is used to adimensionalize the MHD equations, each stellar wind simulation can represent the wind of different stars. We give in Table 1 the magnetic field strength at the base of our models (along with the wind mass and angular momentum loss rates, and the generalized Alfvén radius $\left\langle R_{\mathrm{A}}\right\rangle=\sqrt{\dot{J} / \Omega_{\star} \dot{M}}$ ) for a coronal base density chosen to achieve a solar-like mass loss rate $\left(\rho_{\star}=3.2 \times 10^{-15} \mathrm{~g} \mathrm{~cm}^{-3}\right.$ in the dipolar case and $2.8 \times 10^{-16} \mathrm{~g} \mathrm{~cm}^{-3}$ in the quadrupolar case). In the remainder of this paper, the dimensional quantities are given using these base coronal densities unless stated otherwise.

\subsection{Planet Models}

The planet is modeled as a second internal boundary condition inside the computational domain. We consider here planets in circular orbits with an orbital plane perpendicular to the stellar rotation axis. The MHD equations are solved in a frame rotating at the keplerian orbital rate of the planet $\Omega_{\mathrm{K}}=\sqrt{G M_{\star} / R_{\mathrm{orb}}{ }^{3}}$ (in the limit of a small planetary mass), where $R_{\text {orb }}$ is the orbital radius. The boundary cells composing the star and the planet are fixed in the rotating frame, simplifying the numerical setup significantly.

In this study we set the planetary mass and radius to $M_{P}=0.01 M_{\star}$ and $R_{P}=0.1 R_{\star}$ as in Strugarek et al. (2014b). We also hold fix the orbital radius $R_{\text {orb }}=5 R_{\star}$. We consider planets with an intrinsic dipolar magnetic field oriented along the rotation axis of the host star and simulate three topological situations. Using the dipolar wind solution, we consider the cases of a planet with a dipolar field aligned with the local magnetic field (hereafter the "aligned" case) and of a planet with an anti-aligned dipolar field (hereafter "anti-aligned"). ${ }^{5}$ With the quadrupolar wind solution we simulate a planet with a dipolar field parallel to the stellar rotation axis, and hence perpendicular to the local magnetic field (hereafter the "perpendicular" case). We show the three magnetic configurations in Figure 1, where the field lines of the stellar wind are shown in black and the planetary field lines in red. In the dipolar wind cases the planetary dipole strength corresponds to an equatorial field at the planetary surface $B_{P}=0.9 \mathrm{G}$ (roughly five times less than the jovian magnetic field), and in the quadrupolar wind cases $B_{P}=0.25 \mathrm{G}$. Such planetary fields ensure extended planetary magnetospheres while not constraining the numerical time steps too much.

The planet is initialized at the beginning of the simulations along with the stellar wind (see Section 2.2). We choose to neglect any kind of atmospheric escape to focus our study solely on the magnetic interactions (see Matsakos et al. 2015, for an overview of the impact of atmospheric escape and planetary "winds" in the context of SPMI). The density of the planetary boundary is chosen to be ten times the local wind density, and the pressure to be $90 \%$ of the local thermal pressure of the wind. This ensures that the planet is a cold, dense obstacle in the stellar wind from which no "wind" is triggered. Setting a higher density and/or a lower pressure changes the structure of the planetary magnetosphere very marginally close to the planet boundary, and does not affect our results regarding the interaction between the planetary magnetosphere, the stellar wind, and its host star. The velocity inside the planet and its magnetosphere is initially set to zero in the rotating frame, effectively modeling the orbital motion of the planet and its magnetosphere. We hence consider here only planets that are "tidally locked," which is a reasonable assumption for such close-in planets. The "tidal-locking" timescale for close-in Jupiter-like planets is estimated to be of the order of 0.1-1 Myr (see, e.g., Leconte et al. 2010), which is shorter than the typical migration timescales associated with magnetic torques that we derive in Section 4. We hence consider in this work that the planet is already tidally locked on a circular orbit to focus on the magnetic torques themselves. The velocity, density, and pressure are held fixed during the simulation inside the planet boundary and left free to evolve in the magnetosphere. The magnetic field is maintained as the

\footnotetext{
5 Note that in Strugarek et al. (2014b), the "aligned" and "anti-aligned" denominations had the opposite meaning: they referred to the relative orientation of the planetary and stellar dipoles. In this work we prefer not to use this convention since, for more complex topologies, a denomination based on the local orientation of the magnetic fields in the vicinity of the planet is more intuitive.
} 

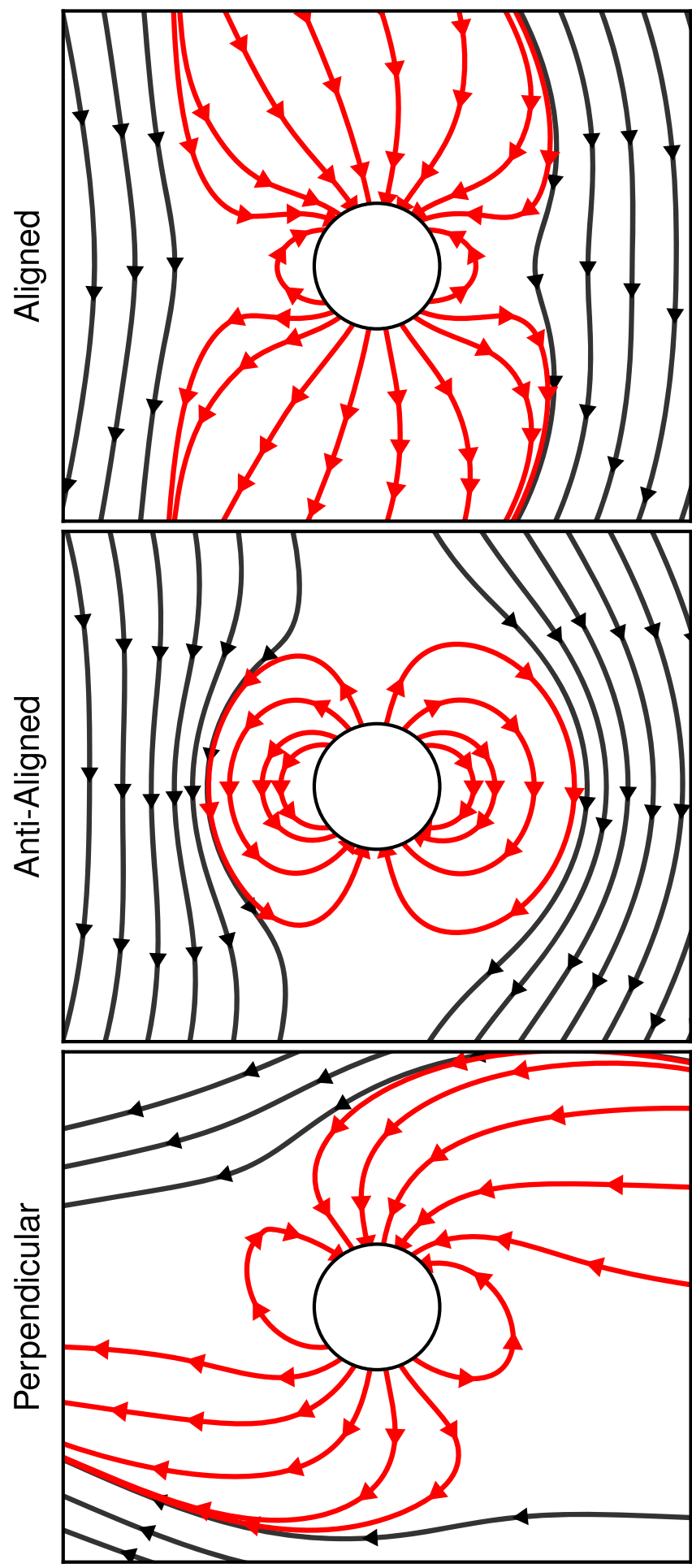

Figure 1. Close-in views of the three magnetic configurations shown in this work. The configurations are labelled by the orientation of the planetary field (in red) with respect to the local stellar wind magnetic field (in black), i.e., aligned, anti-aligned, and perpendicular, from top to bottom.

initial planetary dipole in the inner $70 \%$ of the planet and left free to evolve elsewhere. The planet boundary condition hence possesses an outer layer in which only the magnetic field is allowed to change, crudely mimicking a thick ionosphere. After its initialization, the planetary magnetosphere changes dynamically until a steady state is reached, in which the pressure balance determines the shape of the magnetosphere. In the case we consider here, the total pressure in the planet's vicinity is dominated by the magnetic pressure, which ensures that the magnetic interaction develops in the sub-alfvénic regime.

\subsection{Numerics}

We use the modular PLUTO code (Mignone et al. 2007) to solve the ideal MHD Equations (1)-(6). The equations are solved with a second-order, linear spatial interpolation coupled to the standard HLL Riemann solver and a minmod flux limiter. The variables are updated in time with a second-order RungeKutta method. The solenoidality of the magnetic field is ensured to machine precision with a constrained transport method (Evans \& Hawley 1988), in which the face-centered electromotive forces are arithmetically averaged.

We solve the MHD equations in cartesian geometry with two internal boundary conditions inside the domain, modeling the star (Section 2.2) and the planet (Section 2.3). We recall that the equations are solved in a rotating frame with the rotation rate $\Omega_{\mathrm{K}}$, ensuring that the location of the planet can be held fixed in the simulation grid. At the domain external boundaries we impose simple outflow conditions (zero gradient on all quantities). Because we model stellar winds, the flow is supersonic and super-alfvénic when it reaches the outer boundaries, hence they have little to no impact on the general solution, which is driven by the internal stellar boundary condition.

The simulation are run on a $490 \times 355 \times 355$ cartesian grid. The cube of size $3 R_{\star}$ enclosing the central star is discretized over 97 uniform cells in each direction, and the cube of size $R_{\star}$ enclosing the planet over 161 uniform cells. The remainder of the simulation domain is filled with stretched grids in the three directions toward the domain's limits $\left[-20 R_{\star}, 20 R_{\star}\right]^{3}$.

We stress that we use an ideal set of MHD Equations (Section 2.1), which implies that the only dissipative processes occurring in our simulations are controlled by the numerical scheme and the resolution we choose. This limitation is a reasonable trade-off between numerical simplicity and physical accuracy of our models. Indeed, in all the cases we consider in this work the star-planet system quickly reaches a steady state because the planet is orbiting in a purely axisymmetric wind (see Section 2.2). As a result, the detailed reconnection process between, e.g., the stellar wind and the planetary magnetosphere influences the final steady state only marginally. This is confirmed with additional simulations we ran with halfresolution in the cube of size $R_{\star}$ around the planet, which encloses the main reconnection sites. In these simulations the energy and angular momentum transfers (see Sections 3 and 4) are decreased by less than $15 \%$. Hence, higher resolution, more accurate simulations would lead to slightly stronger magnetic interaction, but not qualitatively change the results presented in this work, as expected. Nevertheless, because we chose an ideal MHD approach, our model is not suited to tackling the dynamical response of the interaction to perturbations or nonaxisymmetric structures in the stellar wind. Hence, we only consider the case of axisymmetric stellar winds in this work. Thanks to PLUTO's modular capabilities, such dynamical processes could be studied more accurately by taking into account explicit ohmic, Hall, and eventually ambipolar dissipation (these effects are not included in the public version of PLUTO yet; for first implementations see Lesur et al. 2014; 
Nakhaei et al. 2014). These aspects are beyond the scope of the present work and will be explored in future studies.

\section{ALFVÉN WINGS}

The concept of Alfvén wings goes back to the pioneering work of Drell et al. (1965), in the context of satellites moving perpendicularly to a uniform magnetic field. Such a satellite excites alfvénic perturbations that propagate along the magnetic field lines, effectively developing currents resembling airplanes' swept-back "wings". We detail in this section how Alfvén wings develop in close-in star-planet systems, and how the magnetic topology affects their shape and characteristics.

\subsection{Structure of the Alfvén Wings}

Alfvén wings develop in close-in star-planet systems due to the differential motion between the orbiting planet and the rotating stellar wind. The orbiting planet excites magnetohydrodynamic perturbations in the stellar wind that propagate along the Alfvén characteristics (Drell et al. 1965; Neubauer 1998; Saur et al. 2013):

$$
\boldsymbol{c}_{\mathrm{A}}^{ \pm} \equiv \boldsymbol{v} \pm \boldsymbol{v}_{\mathrm{A}} .
$$

These perturbations are a vector of electromagnetic energy and angular momentum transport between the planet and its environment, the latter being either the interplanetary medium or the host star. The superposition of the travelling perturbations forms what is referred to as an "Alfvén wing" (Neubauer 1998). If the local Alfvén speed is sufficiently high and the planet is located inside the Alfvén surface of the stellar wind, some of the perturbations can be reflected at the stellar surface and reach back to the orbiting planet. This extreme case is often referred to as the unipolar inductor case, while the case where no perturbations reach back to the planet is called the pure Alfvén wing case. The star-planet system systematically develops two Alfvén wings, along $c_{\mathrm{A}}^{-}$and $c_{\mathrm{A}}^{+}$, located in the $\left(\boldsymbol{v}_{0}, \boldsymbol{B}_{w}\right)$ plane, where $\boldsymbol{B}_{w}$ is the stellar wind magnetic field and $\boldsymbol{v}_{0}=\boldsymbol{v}_{w}-\boldsymbol{v}_{\mathrm{k}}$ is the differential motion between the planet and the wind ( $\boldsymbol{v}_{\mathrm{k}}=R_{\mathrm{orb}} \Omega_{\mathrm{K}} \boldsymbol{e}_{\varphi}$ is the keplerian velocity).

We display in Figure 2 the global structure of the Alfvén wings (right panels) with close-ups on the vicinity of the planet (left panels) for each of three cases we consider. The parallel currents

$$
j_{||}=\boldsymbol{J} \cdot \frac{\boldsymbol{B}}{|\boldsymbol{B}|},
$$

often referred to as Alfvén wing currents (due to the fact that they delimit Aflvén wings, see, e.g., Preusse et al. 2006; Jia et al. 2008), are shown by the red (positive) and blue (negative) volume renderings. These volume renderings are extruded at the vertical star-planet plane to make their internal structure apparent. The magnetic field lines connected to the planet are shown in gray, and the magnetic field lines connected to the star are color-coded by the logarithm of the magnetic field strength. The orbit of the planet is symbolized by the black dashed circle, and the star and the planet are respectively represented by the orange and blue spheres.

In the case of a dipolar stellar magnetic field (upper and middle panels), the two Alfvén wings are connected to the star at high latitude. Conversely, in the quadrupolar stellar wind (lower panels), only one of the two Alfvén wings is connected to the star on the magnetic equator. The footprint of the wings is generally out of phase from the planetary orbital phase due to the finite propagation time of the alfvénic disturbances from the planet's vicinity to the stellar surface (see Preusse et al. 2006; Kopp et al. 2011). In all cases the alfvénic perturbations rapidly travel the planet-star distance along the Alfvén wings in less than $2 \mathrm{hr}$, which is less than $6 \%$ of the orbital time $t_{\text {orb }}=2 \pi R_{\text {orb }} / v_{\mathrm{K}} \sim 1.3$ days. As a result, we observe a small phase lag due to the fast propagation time and the small inclination angle of the wings (see hereafter Equation (9) and Table 2). The travel time is nevertheless always larger than the typical advection time across the planetary diameter $t_{s}=2 R_{P} / v_{\mathrm{K}} \sim 10$ minutes, due to the fast orbital motion of the close-in planet. Hence, these perturbations are likely to never reach back to the orbiting planet and our simulations are always in the regime of pure Alfvén wings. The upper right and middle right panels differ only by an inversion of the planetary magnetic field. We immediately remark the importance of topology: in the aligned case the Alfvén wings are broader and the currents much stronger than in the anti-aligned case. As a result the Alfvén wings are expected to carry much less energy and angular momentum in the anti-aligned case.

In the upper panel of Figure 3 we quantify the parallel currents near the planet. We show a side cut of the planet's vicinity (the star is located leftwards in those cuts), for which the keplerian orbital motion of the planet is into the plane. The black lines represent the magnetic field lines. The parallel currents are again shown by the red (positive) and blue (negative) colormap. They are normalized by $t_{s} / \sqrt{4 \pi \rho}$, thus effectively estimating the ratio between $t_{s}$ and the timescale associated with the parallel currents. The most intense currents are found at the boundary between the planetary magnetic field lines and the wind: they originate from the planet-wind interaction as a simple rotational discontinuity. They are associated with very short timescales compared to the advection timescale $t_{s}$ and trace the-comparatively-fast reconnection rate between the planetary and wind magnetic fields as the planet orbits in the wind. We stress here again that the aligned and anti-aligned cases (two left panels) develop very different interaction patterns. In the aligned case, the strong currents are localized at the boundary between open and closed magnetic field lines and at the Alfvén wing boundaries. In the anti-aligned case, they are mainly localized at the magnetsophere-wind boundary. The perpendicular case shows an in-between situation where the strong currents delimit the Alfvén wing boundaries and the magnetosphere-wind interface.

The Alfvén wings plane $\left(\boldsymbol{v}_{0}, \boldsymbol{B}_{w}\right)$ is shown in the middle panels. The black lines again represent the magnetic field lines, where the thicker lines are the planetary magnetic field lines (the planetary magnetic field lines pervade the Alfvén wings plane in the perpendicular case, since the Alfvén wings plane coincides with the orbital plane in this case). In all cases the stellar wind magnetic field lines are bent downstream due to the interaction with the planet. The difference between aligned and anti-aligned configurations appears clearly: in the aligned case the extended polar magnetic field lines of the planet allow for a large area of magnetic interaction with the wind, whereas in the anti-aligned case the planetary magnetopshere remains in a 


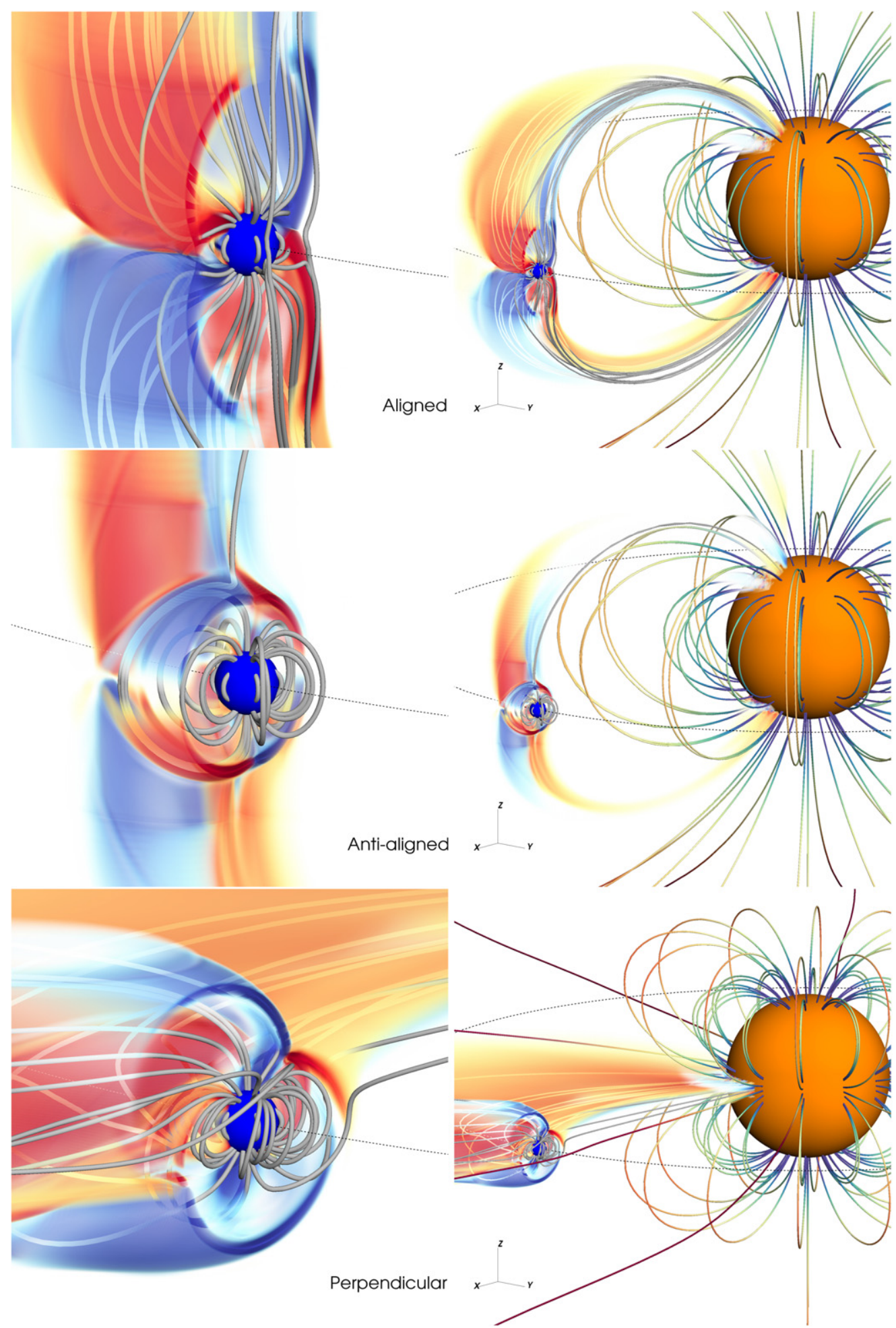

Figure 2. Three-dimensional views of the aligned (top row), anti-aligned (middle row), and perpendicular (bottom row) configurations. The volume renderings represent the postive (red) and negative (blue) parallel currents (Equation (8)) delimiting the Alfvén wings. The volume is extruded from the star-planet plane to make its internal structure apparent. As a result the upstream-downstream asymmetry of the interaction is not visible; it will appear more clearly in Figure 3. The stellar wind magnetic field lines are logarithmically color-coded with the magnetic field strength, and planetary magnetic field lines are shown in gray. The dashed black circle traces the orbit of the planet. The blue sphere represents the planet boundary, and the orange sphere the stellar boundary.

closed configuration. In both cases, though, Alfvén wings develop, symbolized by the blue $\left(c_{\mathrm{A}}^{-}\right)$and red $\left(c_{\mathrm{A}}^{+}\right)$streamlines of the Alfvén characteristics. The expected theoretical inclination angle $\Theta_{\mathrm{A}}$ between the ambient magnetic field and the Alfvén wings is shown by the magenta dashed lines and is given in those cases by (Saur et al. 2013)

$$
\sin \Theta_{\mathrm{A}}^{\mathrm{th}}=\frac{M_{\mathrm{A}} \sin \Theta}{\sqrt{1+M_{\mathrm{A}}^{2}-2 M_{\mathrm{A}} \cos \Theta}},
$$


Table 2

Properties of Alfvén Wings

\begin{tabular}{lccc}
\hline \hline & Aligned & Anti-aligned & Perpendicular \\
\hline$\Theta_{\mathrm{A}}^{\text {th }}(\mathrm{deg})$ & 25 & $\ldots$ & 25 \\
$\Theta_{\mathrm{A}}(\mathrm{deg})$ & 29.7 & 28.2 & 27.3 \\
$R_{\text {eff }}^{\text {th }}\left(R_{P}\right)$ & 3.6 & $\ldots$ & 3.0 \\
$R_{\text {eff }}\left(R_{P}\right)$ & 3.0 & 1.2 & 2.2 \\
$\mathcal{P}^{\text {th }}(\mathrm{W})$ & $2.05 \times 10^{19}$ & $\ldots$ & $1.09 \times 10^{18}$ \\
$\mathcal{P}(\mathrm{W})$ & $1.39 \times 10^{19}$ & $9.74 \times 10^{17}$ & $7.72 \times 10^{17}$ \\
\hline
\end{tabular}

Note. The theoretical th values are estimates from the analytical model of Saur et al. (2013) (in which the anti-aligned case is not modeled).

where the wind Aflvén Mach number is defined by $M_{\mathrm{A}}=v_{0} / v_{\mathrm{A}}$ and $\Theta$ is the angle between $v_{0}$ and $\boldsymbol{B}_{w}$ (note that in Saur et al. $2013 \Theta$ is the departure from perpendicularity between $\boldsymbol{v}_{0}$ and $\boldsymbol{B}_{w}$ ). It is worth noticing that the theoretical inclination angle $\Theta_{A}^{\text {th }}$ is expected to be independent of the planetary magnetic field strength, which is held fixed in our three cases. The theoretical estimate $\Theta_{\mathrm{A}}^{\mathrm{th}}$ compares reasonably well with the simulated inclination angle of the Alfvén wings $\Theta_{\mathrm{A}}$ (averaged over the two wings, the characteristics of the Alfvén wings are summarized in Table 2). The background colormap shows the aziumthal velocity in the frame where the planet is at rest, normalized to the keplerian velocity $v_{\mathrm{K}}$. In all cases the magnetosphere of the planet orbits with the planet (white regions), as well as the portion of the Alfvén wings intersected by the cutting plane.

Finally, in the lower panels we display the flow in the rest frame of the planet (blue streamlines) and the plasma density (logarithmic colormap, normalized to the planet's density) on the planetary orbital plane. In the first two panels the flow is primarily in the orbital direction because the planet orbits inside the dead-zone of the stellar corona, where the radial flow of the stellar wind is negligible. The effective obstacle is larger on the equatorial plane in the anti-aligned case due its larger magnetospheric extent (this is also apparent in the middle panels). The effective obstacle is nevertheless three-dimensional and is generally much bigger in the aligned case (see Section 4). No strong wake is observed downstream since we chose to neglect planetary outflows and the fact that the interaction is subalfvénic. In the third panel, the stellar wind magnetic field lines are open in the orbital plane. The flow is consequently composed of the orbital motion and the accelerating radial wind. The planetary obstacle is observed to be much larger than the planetary magnetosphere. Indeed, the Alfvén wings extend toward and away from the star on the orbital plane and act as a supplementary obstacle to the flow (see also the $v_{\varphi}$ colormaps in the middle panels). Since we considered the idealized case of a planet with no intrinsic mass loss, the high planet density does not propagate to more than a few grid points inside the planet magnetosphere. Varying the planet's internal density only marginally affects our results. The detailed density pattern in the planetary magnetosphere is found to have very little impact on the properties of the Alfvén wings.

\subsection{Poynting Flux in Alfvén Wings}

The magnetic interaction is a source of magnetic energy transfer between the wind, the planet, and the host star. It could be a source of observable emissions, and thus its characterization is of major importance to the search for exoplanets today. The Poynting flux in each Alfvén wing can be evaluated as

$$
S_{a}=\frac{c \boldsymbol{E} \times \boldsymbol{B}}{4 \pi} \cdot \frac{\boldsymbol{c}_{\mathrm{A}}^{ \pm}}{\left|\boldsymbol{c}_{\mathrm{A}}^{ \pm}\right|},
$$

where the electric field is $c \boldsymbol{E}=-\boldsymbol{v} \times \boldsymbol{B}$ in the ideal MHD approximation. The Poynting flux depends on the frame in which it is calculated; as a result we consider here the inertial reference frame to mimic what a distant observer would see when observing such a system. Because the central star rotates slowly, this frame also conveniently corresponds to the stellar reference frame, and the Poynting flux corresponds to the energy that may be deposited on the star due to the SPMI. We display in Figure 4 the Poynting flux on horizontal cutting planes along the $c_{\mathrm{A}}^{-}$Alfvén wing for the aligned and anti-aligned cases, slightly above the equatorial plane at $z=0.3,0.5,0.7 R_{\star}$. For the quadrupolar stellar wind (right panels) the Alfvén wings are centered on the orbital plane, hence we display the Poynting flux along the $c_{\mathrm{A}}^{+}$Alfvén wing on vertical cutting planes in between the planet and the star. In each panel, the blue circle and the dashed line respectively represent the projection of the planet and that of its orbital trajectory on the cutting plane.

Comparing the aligned and anti-aligned cases (left two panels), we immediately observe the strong Poynting flux concentrated inside the Alfvén wing in the aligned case, while in the anti-aligned case the Poynting flux is extremely weak. In all cases the flux is nevertheless positive, denoting a flux of energy toward the star. In the aligned and perpendicular cases the Alfvén wing is tear-shaped in the direction of the flow (gray arrows), and the maximum Poynting flux is localized close to the side of the wing facing the flow. In these two cases the Alfvén wing clearly acts as an obstacle to the flow. Conversely, in the anti-aligned case, the very small cross-section of the Alfvén wing makes only a small perturbation to the flow, as seen in the middle panels. As the cutting plane is shifted away from the planet (from top to bottom), the center of the Alfvén wing is observed to shift toward the star and downstream.

The cross-section of the Alfvén wing perpendicular to the flow, $2 R_{\text {eff }}$, is indicated by the double arrows in Figure 4 , and computed by taking the maximal extent of the wing perpendicular to the flow. It remains approximately constant along the wing as long as the cutting plane remains roughly perpendicular. Saur et al. (2013) used a simple magnetostatic equilibrium code to estimate the expected $R_{\text {eff }}$ as a function of the standard obstacle radius $R_{\text {obst }}$ (e.g., Lovelace et al. 2008; Lanza 2009), which are defined by

$$
\begin{gathered}
R_{\mathrm{eff}} \sim R_{\mathrm{obst}}\left(3 \cos \frac{\Theta_{M}}{2}\right)^{1 / 2}, \\
R_{\mathrm{obst}} \sim R_{p}\left(\frac{B_{P}^{2}}{8 \pi P_{t}}\right)^{1 / 6},
\end{gathered}
$$

where $P_{t}$ is the total pressure in the vicinity of the planet, and $\Theta_{M}$ is the inclination angle between the polar planetary field and $\boldsymbol{B}_{w}$ (we focus here on the case of a magnetized planet; the effective obstacle in the case of a planet with no intrinsic magnetism is discussed, e.g., by Kopp et al. 2011). Because of the magnetostatic equilibrium assumed to derive Equation (11), 

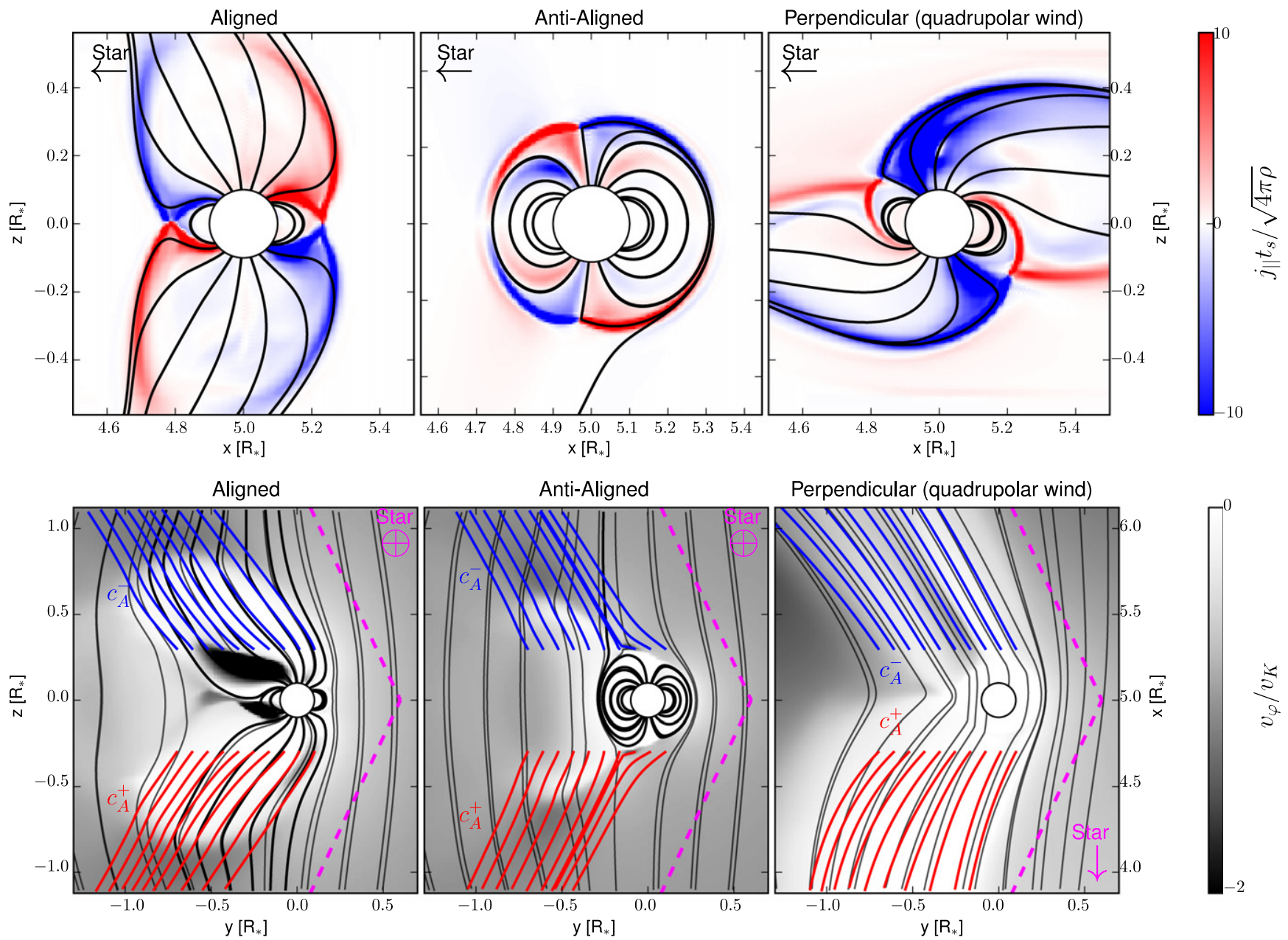

Perpendicular (quadrupolar wind)
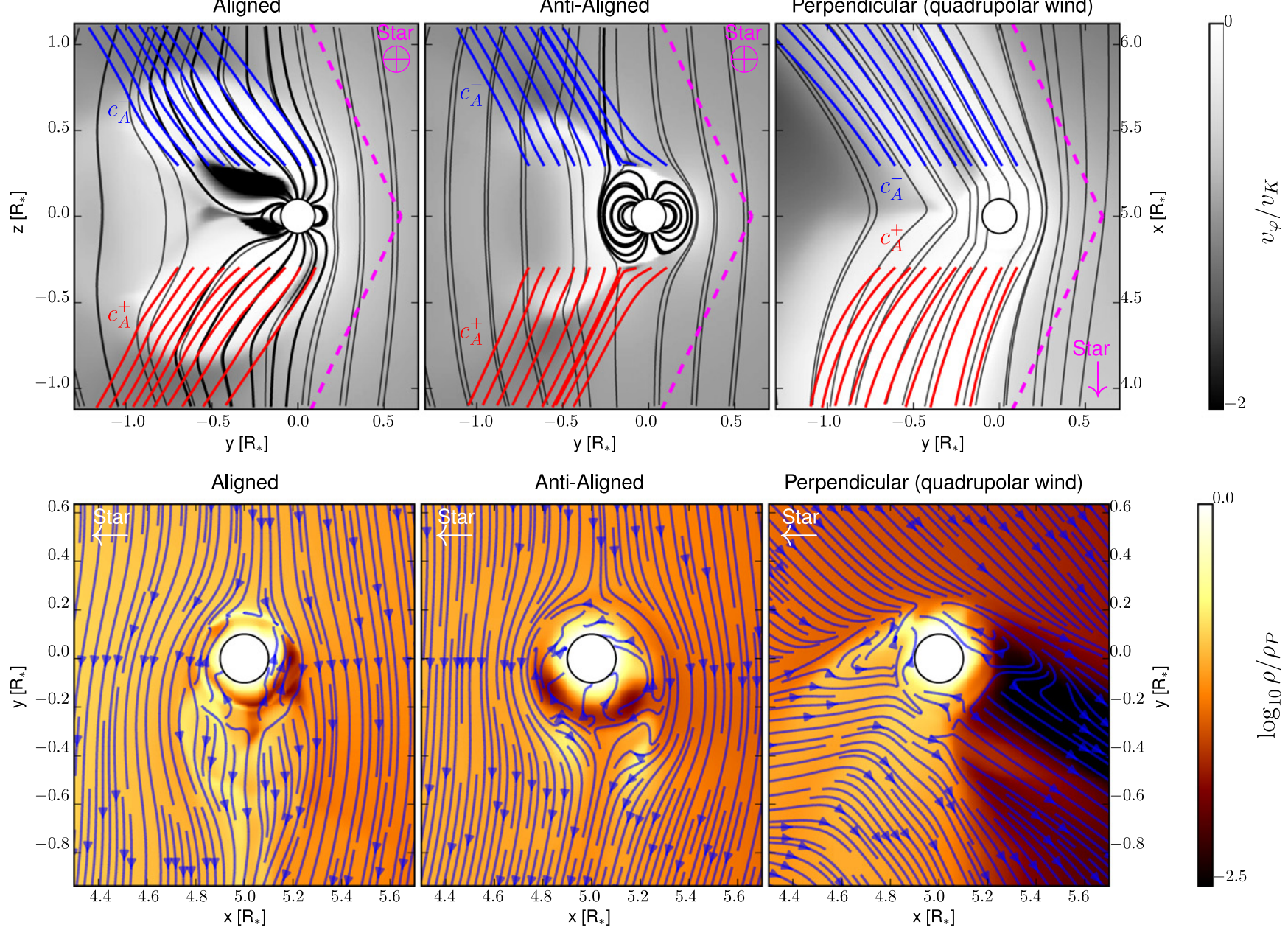

Figure 3. Top: positive (red) and negative (blue) parallel currents in the star-planet plane perpendicular to the orbital plane. The currents are normalized with the advection timescale across the planet (see text). The black lines represent the magnetic field lines and the white circle represents the planet. Middle: cuts of the $\left(\boldsymbol{v}_{0}, \boldsymbol{B}_{w}\right)$ plane. The gray shades show the azimuthal velocity in the rest frame of the planet, normalized by the keplerian velocity $\boldsymbol{v}_{\mathrm{K}}$. The Alfvén characteristic streamlines away from the planet are shown in blue and red. We do not plot the Alfvén characteristics inside the planetary magnetosphere, where they do not correspond to the travelling path of the perturbations forming the Alfvén wings. The expected inclination angle $\Theta_{\mathrm{A}}^{\text {th }}$ of the Alfvén wings is shown by the purple dashed line. Bottom: density (on a logarithmic scale) in the orbital plane close to the planet. The streamlines of the flow in the orbital plane are shown in blue.

we do not expect it to exactly match our observed effective radii $R_{\text {eff }}$. The estimated and simulated effective radii $R_{\text {eff }}$ are given in Table 2. It appears that the theoretical value slightly overestimates the effective radius we obtain in our simulation. The shape of the simulated Alfvén wing cross-section is much more elongated-in the flow direction-than the theoretical Alfvén wing of Saur et al. (2013). As a result, this effect compensates the discrepancy in the effective radii of the obstacle, and the theoretical and simulated wings have similar cross-sectional areas. 


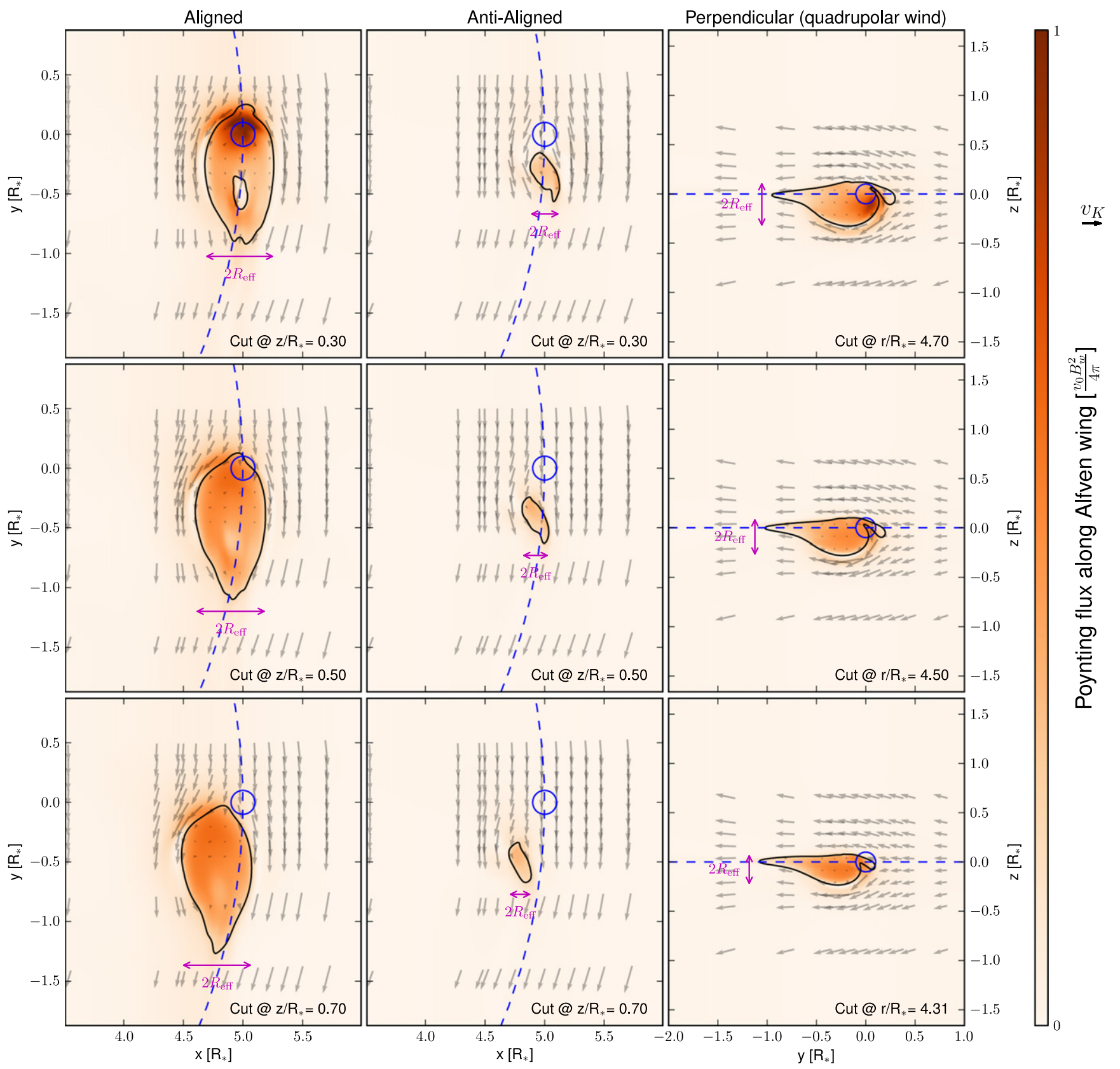

Figure 4. Poynting flux along Alfvén wings. The rows correspond to cuts of the Alfvén wing at distances of $0.3,0.5$, and $0.7 R_{\star}$ from the planet. The Poynting flux is normalized to the maximum expected Poynting flux $v_{0} B_{w}^{2} / 4 \pi$. The black contours represent the boundary of the Alfvén wing, identified as the region co-orbiting with the planet in the projection plane. The cross-section of the Alfvén wing perpendicular to the flow is indicated in purple. The black arrows represent the flow on the cutting plane in the rest frame of the planet. The dashed blue line is the projection of the orbital trajectory and the blue circle the projection of the planet's boundary.

The maximum amplitude of the Poynting flux scales remarkably well with the predicted value of $v_{0} B_{w}^{2} / 4 \pi$ expected from the analytical estimates of Saur et al. (2013). By integrating the total Poynting flux inside the area $\Sigma_{\mathrm{A}}$ delimited by black contours in Figure 4 (effectively delimiting the Alfvén wing cross-section), we find that the total Poynting flux is close to being constant throughout each wing. We report the average value of the integrated Poynting flux

$$
\mathcal{P}=\left\langle\int S_{\mathrm{A}} d \Sigma_{\mathrm{A}}\right\rangle_{\mathrm{A}}
$$

in Table 2, where \langle\rangle$_{\mathrm{A}}$ stands for an average along the Alfvén wing. The theoretical and simulated Poynting fluxes agree within a factor of two, which is satisfying given the approximations embedded in both the analytical and numerical models. The total Poynting flux is smaller by a factor of six between the aligned and the anti-aligned cases. This topological effect could provide a simple explanation for the on/off enhanced emissions observed in extreme exo-systems over the timescales of typical stellar magnetic cycles or orbital periods.

\section{PLANET MIGRATION DUE TO MAGNETIC TORQUES}

We have demonstrated that our numerical model is able to simulate adequately Alfvén wings that compare well with estimates from analytical theory. We now focus on the less 

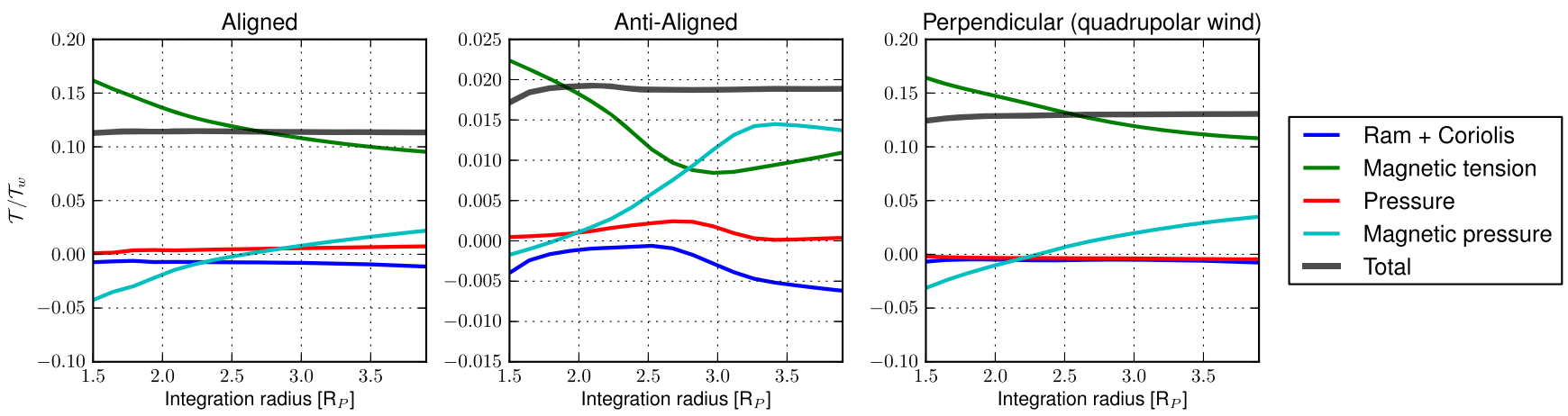

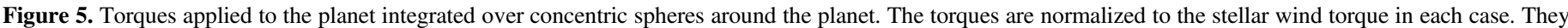

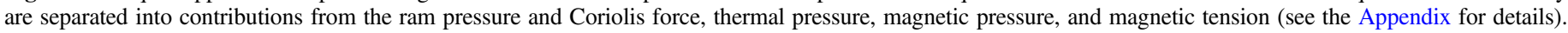
The total torque is indicated in black.

studied magnetic torques, which can play a role in the secular evolution of close-in star-planet systems.

\subsection{Physical Origin of the Torques}

Torques in magnetic star-planet systems can be separated into contributions from ram pressure, thermal pressure, magnetic pressure, and magnetic tension. We derive the angular momentum fluxes associated with those torques in a frame rotating at the planetary orbital rate. The angular momentum is defined with respect to the stellar rotation axis, which coincides with the orbital axis for the cases considered in this work. The interested reader will find the derivation of the various torque expressions in the Appendix.

Thanks to angular momentum conservation, these torques can be evaluated on any surface enclosing the planet. As a result we estimate the torques from integrations over concentric spheres around the planet, and check a posteriori that the total torque $\mathcal{T}$ (black lines in Figure 5) is indeed constant with the integration radius. The different components of the torque are detailed in Figure 5.

In the aligned and perpendicular cases (left and right panels), the torque is dominated by the tension of the magnetic field lines connecting the planet to the star (green line). It is slightly opposed by the magnetic pressure (cyan line) while the ram and thermal pressures play almost no role in the overall torque. Note that in the three cases, the magnetic pressure (cyan) changes sign as the integration sphere surrounding the planet is expanded. This is a simple consequence of the fact that the magnetosphere of the planet is more extended downstream than upstream. As a result, the integration is imbalanced toward the downstream contribution and gives a negative contribution when the integration spheres are fully inside the planetary magnetosphere. When the integration sphere is fully outside the planetary magnetosphere, the contribution of magnetic pressure to the torque is positive, as expected.

In the anti-aligned case (middle panel), the total torque is the combination of both magnetic tension and pressure, corresponding to the wind magnetic field lines impacting the closed planetary magnetosphere. The ram and thermal pressures play a marginal role here as well in the total torque. The torque applied to the planet in the aligned case is roughly $10 \%$ of the stellar wind torque applying to the host star. Because the two Alfvén wings connect back to the star, it means that the star brakes $10 \%$ less efficiently than a twin star not harbouring any close-in planet. The torque in the aligned case is furthermore approximately five times higher than in the anti-aligned case,
Table 3

Torques and Effective Obstacle Areas of the Magnetic Interaction

\begin{tabular}{lccc}
\hline \hline & Aligned & Anti-aligned & Perpendicular \\
\hline $\mathcal{T}\left(\mathcal{T}_{w}\right)$ & 0.11 & 0.02 & 0.13 \\
$c_{d}$ & 0.43 & 1.0 & 0.64 \\
$A_{\text {obst }}^{\text {th }}\left(\pi R_{p}^{2}\right)$ & 4.4 & 4.4 & 4.0 \\
$A_{\text {obst }}\left(\pi R_{p}^{2}\right)$ & 72.4 & 5.1 & 25.0 \\
$t_{P}(\mathrm{Myr})$ & $1.39 \times 10^{2}$ & $8.46 \times 10^{2}$ & $2.70 \times 10^{3}$ \\
\hline
\end{tabular}

Note. The theoretical area $A_{\mathrm{obst}}^{\text {th }}$ is obtained with the expected magnetospheric size $R_{\text {obst }}^{\text {th }}$ from Equation (12). The migration timescale $t_{P}$ is calculated with a base density $\rho_{\star}=3.2 \times 10^{-10} \mathrm{~g} \mathrm{~cm}^{-3}$ for the aligned and anti-aligned cases, and $\rho_{\star}=2.8 \times 10^{-11} \mathrm{~g} \mathrm{~cm}^{-3}$ for the perpendicular case (see the text).

showing again the very strong impact of the magnetic topology on the strength of the SPMI. The perpendicular case shows a very similar repartition of the different contributions to the aligned cases, with a slightly higher magnetic pressure. It is interesting to note that in the perpendicular case only one wing connects the star and the planet; as a result only half of the angular momentum extracted from the orbit of the planet will be transferred to the star, while the other half will be advected in the wind. Hence, the impact of the SPMI on the host star is maximized in the aligned case.

We further calculate the migration timescale associated with those torques, given by

$$
t_{P}=\frac{2 J_{P}}{\mathcal{T}}
$$

where $J_{P}=M_{P}\left(G M_{\star} R_{\text {orb }}\right)^{1 / 2}$ is the orbital angular momentum of the planet, and the factor 2 originates from the $R_{\text {orb }}{ }^{1 / 2}$ dependence of $J_{P}$. The migration timescale depends on the density normalization $\rho_{\star}$. In Table 3 we give the migration timescales (as well as the average torques $\mathcal{T}$ ) for a $\rho_{\star}$ normalization corresponding to the mass loss rate of a $\mathrm{T}$ Tauri star (five orders of magnitude higher than the Sun, corresponding as well to a much stronger stellar magnetic field). The timescales are clearly sufficiently short to suggest that magnetic interactions can play a role in the migration of close-in planets, especially during the early stages (typically $\mathrm{T}$ Tauri and premain sequence phases) of star-planet systems. The density normalization divides the migration timescale $t_{P}$ when computing it from adimensionalized units. For a solar-like density normalization (see Section 2.2), the timescales would 
be five orders of magnitude higher and be negligible compared to typical tidal effects. Preliminary scaling laws for the migration timescales of close-in planets were derived Strugarek et al. (2014b) from reduced 2.5D simulations. The variation of $t_{P}$ with topology in our 3D models agrees with the predicted law from Strugarek et al. (2014b), and the dependence on orbital radius is not expected to change significantly from $2.5 \mathrm{D}$ to $3 \mathrm{D}$ models. Nevertheless, the torques differ qualitatively because the multiplicative constant in front of the scaling law derived in Strugarek et al. (2014b) was calibrated with $2.5 \mathrm{D}$ models. By using a grid of 3D numerical simulations (currently under investigation), we intend to better constrain this multiplicative constant to obtain a quantitatively accurate scaling law in the near future. This extended set of simulations will also confirm the dependence of the migration timescale $t_{P}$ on the orbital radius $R_{\text {orb }}$ found in Strugarek et al. (2014b). Finally, close-in star-planet systems can also be in a super-alfvénic interaction regime (not explored here). Indeed, even if the wind in the vicinity of the planet is sub-alfvénic, the relatively fast orbital motion can exceed the local Alfvén speed. How the torque scaling law changes between the sub- and super-alvénic regimes still remains to be explored.

\subsection{Parameterization of Magnetic Torques and Effects of Magnetic Topology}

By analogy with an obstacle in a flow, the magnetic torque applied to the planet due to the SPMI is generally written as (e.g., Lovelace et al. 2008; Vidotto et al. 2009)

$$
\mathcal{T}=c_{d} R_{\text {orb }} A_{\text {obst }} P_{t},
$$

where $A_{\text {obst }}$ is the effective obstacle area exposed to the flow, $P_{t}$ the total (thermal plus ram plus magnetic) pressure of the wind in the frame where the planet is at rest, and $c_{d}$ a drag coefficient. The right-hand side is conveniently composed of the total angular momentum that can be transferred, multiplied by $c_{d}$. In the case of SPMI, the drag coefficient $c_{d}$ and the effective area $A_{\text {obst }}$ should generally depend on the topology of the interaction, i.e., on the respective orientations of the orbital motion, the interplanetary magnetic field, and the planetary magnetic field. Due to this complexity, the drag coefficient $c_{d}$ and the effective interaction area $A_{\text {obst }}$ can be non-trivial to estimate.

The drag coefficient is generally thought to represent-in the case of SPMI-the reconnection efficiency between the stellar wind and the planetary magnetic fields, at the boundaries of the planetary magnetosphere or of the Alfvén wings themselves. In the context of planetary radio emissions, Zarka (2007) approximated $c_{d}$ with (see also Neubauer 1998; Saur et al. 2013)

$$
c_{d} \sim \frac{M_{\mathrm{A}}}{\sqrt{1+M_{\mathrm{A}}^{2}-2 M_{\mathrm{A}} \cos \Theta}} .
$$

This latter equation is not thought to be valid in the closed magnetosphere case (here the so-called "anti-aligned" case), for which we simply choose $c_{d} \sim 1$. The drag coefficient in the aligned and perpendicular cases is given in Table 3 .

The obstacle area $A_{\text {obst }}$ is generally considered as a circular cross-section of the planetary magnetosphere, of estimated radius $R_{\text {obst }}$ (Equation (12)), itself deduced from a simple pressure balance. However, it is not often recognized that this effective area changes drastically with the magnetic topology and is, in general, far from being circular. We use here our numerical simulations to estimate $A_{\text {obst }}$, based on the integrated torque $\mathcal{T}$ shown in Figure 5. The resulting areas are given in Table 3, along with the standard obstacle area $A_{\mathrm{obst}}^{\mathrm{th}}=\pi R_{\mathrm{obst}}{ }^{2}$. The anti-aligned case (middle panels in Figure 3 ) is the only situation in which the effective obstacle is indeed the roughly circular magnetospheric cross-section perpendicular to the flow (see also schematic in Figure 6).

In the aligned and perpendicular cases (upper and lower panels in Figure 3), the connection between the planetary field and the wind magnetic field leads to an interaction crosssection composed of the whole flux-tube connecting the star and the planet (the width of which is given by $R_{\mathrm{eff}}$, see Table 2), and hence to a much greater torque (as seen in Figure 5). The corresponding obstacle area is found to be 14 times higher than the standard obstacle area in the aligned case, and 5 times higher in the perpendicular case (note that the corresponding torques in Table 3 are normalized to the stellar wind torques $\mathcal{T}_{w}$, which differ in the dipolar and quadrupolar cases as seen in Table 1). The torque is maximized and minimized in the two extreme cases of aligned and anti-aligned topologies. As a result, since we generally cannot infer the magnetic field of known exoplanets, these two cases give good upper and lower estimates of magnetic torques that a given star-planet system can develop.

\section{CONCLUSIONS}

In this work we have simulated in three dimensions the magnetic interactions of a star with a close-in planet. By simulating the system globally, we were able to trace Alfvén wings extending from the planet's magnetosphere to the stellar lower corona. We have explored three typical magnetic configurations of SPMI: aligned, anti-aligned, and perpendicular orientations of the planetary field with respect to the ambient wind magnetic field. For the perpendicular case we chose to consider a dipolar planetary field perpendicular to the orbital plane and a quadrupolar stellar wind. In this latter case the accelerating wind participates in the interaction and the Alfvén wings extend near the orbital plane. In the aligned and anti-aligned cases, the planet orbits inside a wind "dead-zone" and the Alfvén wings extend out of the orbital plane along the dipolar structure of the stellar corona.

The Poynting flux in Alfvén wings provides an energy source for enhanced X-ray or UV emissions in the star-planet system. We were able to validate our numerical model by comparing the simulated Poynting fluxes with analytical predictions. We find that the size of the Alfvén wings (and their associated Poynting flux) depends dramatically on the magnetic configuration of the interaction: by reversing the planetary field, the Poynting flux drops by a factor of approximately 14 .

We used numerical simulations to estimate the magnetic torques that develop in SPMI. Again, the magnetic configuration significantly affects the torques that develop in such systems. In the aligned case, a part of the planetary magnetosphere is open in the ambient stellar wind and connects the star and the planet together. They are a source of magnetic tension that effectively transfers angular momentum between the star and the planet. In the cases presented in this paper, the planet loses orbital angular momentum and migrates inward (for fast 

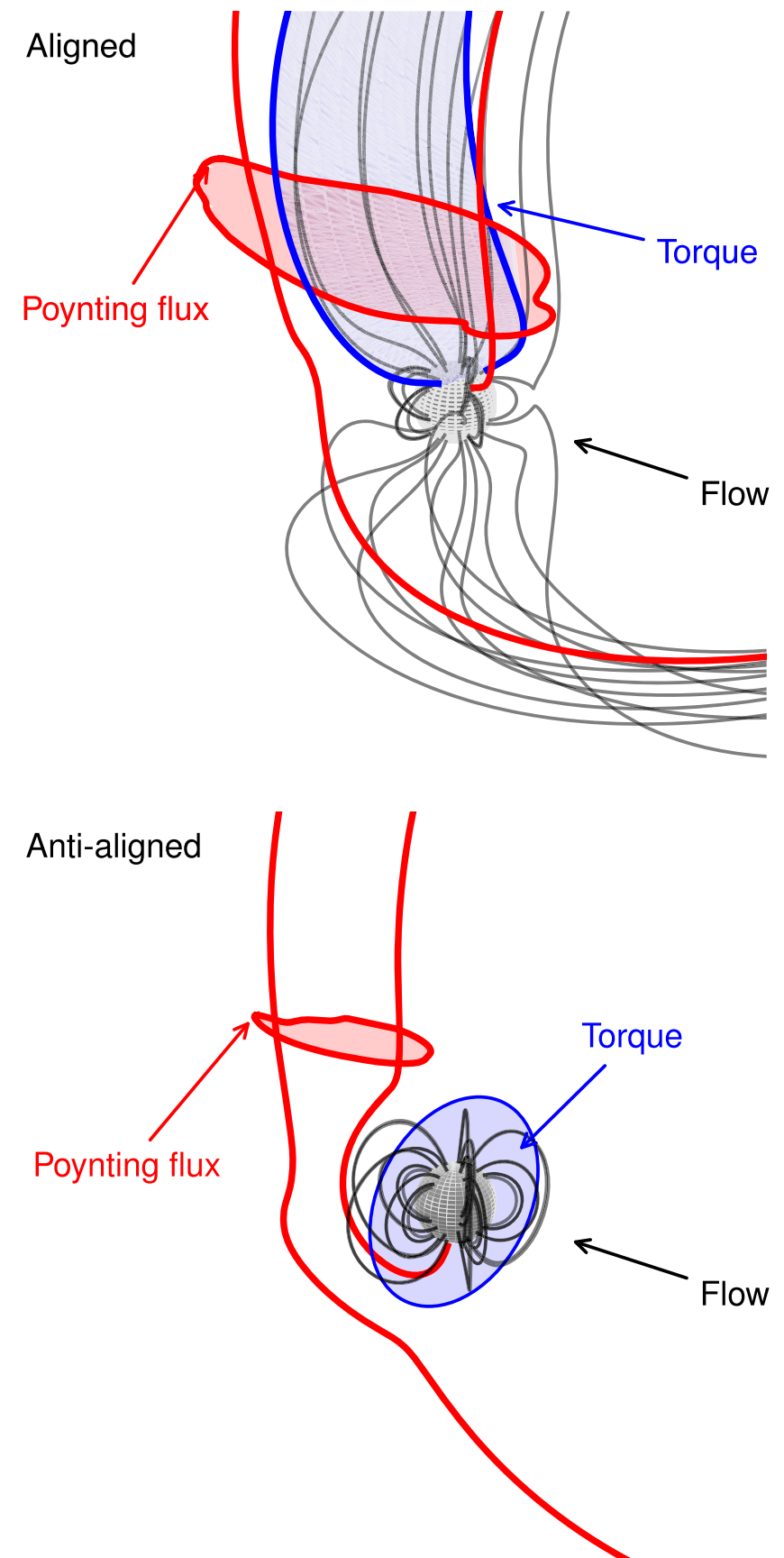

Figure 6. Schematics of magnetic star-planet interactions in the aligned (top) and anti-aligned (bottom) cases. The black lines represent the magnetic field lines; the blue and red lines delimit the upper Alfvén wing. Characteristic surfaces associated with the Poynting flux (red area) and the magnetic torques (blue area) are highlighted in each configuration, showing the critical role of magnetic topology in the development of the interaction.

rotators a planet may a priori gain orbital angular momentum from such interaction; for a detailed discussion see Strugarek et al. 2014b). In the aligned case the migration timescale of the studied planets varies from 100 to $1000 \mathrm{Myr}$ for a T Tauri-like host star. As a result, magnetic interactions can be an important factor in migration for young close-in planets. In the antialigned case the magnetosphere is completely closed: the torque then originates from the coronal plasma impacting on the cross-section of the magnetosphere. The area of interaction is hence much smaller than in the aligned case, and the associated magnetic torque is roughly 14 times smaller.

We illustrate the importance of magnetic configuration in Figure 6 where the aligned and anti-aligned configurations are schematized. The red areas show the cross-section of one Alfvén wing that carries the induced Poynting flux. The blue areas represent the interaction areas leading to magnetic torques. The standard estimation of magnetic torques, based on Equation (15), generally makes use of the effective area of the anti-aligned interaction. We find here that this area minimizes the magnetic torque that can develop in close-in star-planet systems, while the blue area of the aligned case maximizes it.

The three-dimensional simulations reported in this work confirm the 2.5D axisymmetric results of Strugarek et al. (2014b): magnetic torques can be a source of close-in planet migration. We have focused on characterizing the impact of magnetic configuration on the shape and strength of the magnetic interactions. We are currently running a more extensive set of models to empirically refine the scaling laws first derived in Strugarek et al. (2014b).

Real stars possess much more complex magnetic fields than the simple dipolar and quadrupolar configurations we considered in this work. In reality close-in planets are likely to interact with different local magnetic configurations along their orbit (see, e.g., Cohen et al. 2014; Strugarek et al. 2014c; Vidotto et al. 2015). Our results suggest that, in such systems, the associated Poynting fluxes and torques will vary by at least an order of magnitude, which provides a simple geometrical explanation for an on/off mechanism of magnetically enhanced emissions in close-in star-planet systems. The average Poynting flux and torque that such systems develop are nonetheless non-trivial to estimate. They will require dedicated 3D simulations tackling the dynamical aspects of magnetic interactions as a planet orbits in a non-homogenous corona. Indeed, the timescale on which the equilibrated configurations modeled in this paper become established depends on the resistivity of the magnetospheric plasma of the planet, and on its efficiency of reconnection with the stellar wind magnetic field. The numerical model presented in this work provides a solid basis for further, more realistic studies of SPMI in which these dynamical aspects could be explored.

A. Strugarek is a National Postdoctoral Fellow at the Canadian Institute of Theoretical Astrophysics, and acknowledges support from Canada's Natural Sciences and Engineering Research Council. This work was supported by the ANR 2011 Blanc Toupies and the ERC project STARS2. We acknowledge access to supercomputers through GENCI (project 1623), Prace (8th call), and ComputeCanada infrastructures.

\section{APPENDIX GENERAL EXPRESSIONS OF TORQUES}

We derive here the general expression for torques in a rotating frame, based on the initial derivation of Mestel \& Selley (1970). The momentum equation in the MHD formalism in a rotating frame (with a rotation rate $\Omega$ ) can be written as

$$
\begin{aligned}
\partial_{t}(\rho \boldsymbol{u})= & \boldsymbol{\nabla} \cdot\left(-\rho \boldsymbol{u} \boldsymbol{u}+\boldsymbol{B B} / 4 \pi-\boldsymbol{I} P_{t}\right) \\
& +\rho \boldsymbol{g}-2 \rho \boldsymbol{\Omega} \times \boldsymbol{u}+\rho \boldsymbol{\Omega} \times \boldsymbol{\Omega} \times \boldsymbol{r},
\end{aligned}
$$


where the total pressure is the sum of the thermal pressure and the magnetic pressure $P_{t}=P+B^{2} / 8 \pi$. We define the vectorial angular momentum by

$$
\mathcal{J} \equiv \int_{V} \boldsymbol{r} \times \rho \boldsymbol{u} d V
$$

with $V$ a given volume. The time evolution of the angular momentum, or equivalently the torques acting on the volume $V$, is given by

$$
\dot{\mathcal{J}}=\int_{V} \boldsymbol{r} \times \partial_{t}(\rho \boldsymbol{u})+\partial_{t} \boldsymbol{r} \times \rho \boldsymbol{u} d V=\int_{V} \boldsymbol{r} \times \partial_{t}(\rho \boldsymbol{u}) d V,
$$

where we supposed that the volume $V$ is held constant in the rotating frame.

In the context of star-planet systems, we are here primarily interested in the rotational angular momentum of the star and in the orbital angular momentum of the planet. Both are defined through Equation (18), respectively projected on the rotation axis and on the normal to the orbital plane.

Combining Equations (19) and (17) we get

$$
\begin{aligned}
\dot{\mathcal{J}}= & \int_{V} \boldsymbol{r} \times\left[\boldsymbol{\nabla} \cdot\left(-\rho \boldsymbol{u} \boldsymbol{u}+\boldsymbol{B B} / 4 \pi-\boldsymbol{I} P_{t}\right)\right] d V-2 \\
& \times \int_{V} \rho \boldsymbol{r} \times(\boldsymbol{\Omega} \times \boldsymbol{u}) d V \\
& +\int_{V} \rho \boldsymbol{r} \times(\boldsymbol{\Omega} \times(\boldsymbol{\Omega} \times \boldsymbol{r})) d V+\int_{V} \rho \boldsymbol{r} \times \boldsymbol{g} d V .
\end{aligned}
$$

In all the cases considered here, the gravity profile will be close to symmetric in the volume of integration $V$, hence the last term of Equation (20) will be neglected. The centrifugal contribution can be reworked through

$$
\begin{array}{r}
\int_{V} \rho \boldsymbol{r} \times(\boldsymbol{\Omega} \times(\boldsymbol{\Omega} \times \boldsymbol{r})) d V= \\
\int_{V} \rho \boldsymbol{\Omega} \times(\boldsymbol{r} \times(\boldsymbol{\Omega} \times \boldsymbol{r})) d V .
\end{array}
$$

In order to rewrite the first two terms on the right-hand side of (20), we use the Levi-Civita permutation symbol $\varepsilon_{i j k}$ and Einstein's summation notation $\left((\boldsymbol{a} \times \boldsymbol{b})_{i}=\varepsilon_{i j k} a^{j} b^{k}\right)$. If a tensor $\boldsymbol{C}$ is symmetric, we can write

$$
\partial_{x_{l}}\left(\varepsilon_{i j k} x_{j} C_{k l}\right)=\varepsilon_{i j k}\left(C_{k l} \delta_{j l}+x_{j} \partial_{x_{l}} C_{k l}\right)=\varepsilon_{i j k} x_{j} \partial_{x_{l}} C_{k l} .
$$

Since the three tensors in the first term on the right-hand side of (20) are symmetric, we can define $\boldsymbol{T} \equiv-\rho \boldsymbol{u} \boldsymbol{u}+\boldsymbol{B} \boldsymbol{B}-\boldsymbol{I} P_{t}$ and write

$$
\begin{aligned}
{\left[\int_{V} \boldsymbol{r} \times[\boldsymbol{\nabla} \cdot \boldsymbol{T}] d V\right]_{i} } & =\int_{V} \varepsilon_{i j k} x_{j} \partial_{x_{l}} T_{k l} d V \\
& =\int_{V} \partial_{x_{l}}\left(\varepsilon_{i j k} x_{j} T_{k l}\right) d V \\
& =\int_{S} \varepsilon_{i j k} x_{j} T_{k l} n_{l} d S,
\end{aligned}
$$

where $S$ is the surface bounding $V$ and $\boldsymbol{n}$ the normal to this surface. Finally, we expand the Coriolis contribution by conveniently introducing the following surface integral:

$$
\begin{aligned}
A_{i} & \equiv \int_{S}[\boldsymbol{r} \times(\boldsymbol{\Omega} \times \boldsymbol{r})]_{i}(\rho \boldsymbol{u} \cdot d \boldsymbol{S}) \\
& =\int_{S} \varepsilon_{i j k} x_{j} \rho u_{l} \varepsilon_{k m n} \Omega_{m} x_{n} n_{l} d S \\
& =\int_{V} \partial_{x_{l}}\left(\varepsilon_{i j k} \varepsilon_{k m n} \rho x_{j} u_{l} \Omega_{m} x_{n}\right) d V \\
& =\int_{V} \rho u_{l} \partial_{x_{l}}\left(\left[\delta_{i m} \delta_{j n}-\delta_{j m} \delta_{i n}\right] \Omega_{m} x_{j} x_{n}\right) d V \\
& =\int_{V} \rho u_{l} \partial_{x_{l}}\left(\Omega_{i} x_{n} x_{n}-\Omega_{j} x_{j} x_{i}\right) d V \\
& =\int_{V} \rho\left(2 \Omega_{i} x_{n} u_{n}-\Omega_{j} x_{j} u_{i}-x_{i} \Omega_{j} u_{j}\right) d V,
\end{aligned}
$$

where we have used the mass continuity equation $\partial_{x_{l}}\left(\rho u_{l}\right)=0$ that is satisfied in a steady state. We note that the Coriolis contribution in Equation (20) can be written as

$$
\begin{aligned}
\mathcal{C}_{i} & \equiv\left[-2 \int_{V} \rho \boldsymbol{r} \times(\boldsymbol{\Omega} \times \boldsymbol{u}) d V\right]_{i} \\
& =\int_{V}-2 \rho \varepsilon_{i j k} x_{j} \varepsilon_{k m n} \Omega_{m} u_{n} d V \\
& =\int_{V}-2 \rho\left(\delta_{i m} \delta_{j n}-\delta_{j m} \delta_{i n}\right) \Omega_{m} u_{n} x_{j} d V \\
& =\int_{V} \rho\left(2 \Omega_{j} x_{j} u_{i}-2 \Omega_{i} u_{j} x_{j}\right) d V .
\end{aligned}
$$

Combining Equations (23) and (24) we obtain

$$
\begin{aligned}
\mathcal{C}_{i} & =-A_{i}+\int_{V} \rho\left(u_{i} \Omega_{j} x_{j}-x_{i} \Omega_{j} u_{j}\right) d V \\
d V & =-A_{i}+\left[\int_{V} \rho \boldsymbol{\Omega} \times(\boldsymbol{r} \times \boldsymbol{u})\right]_{i} .
\end{aligned}
$$

We can finally combine Equations (20), (21), and (25) to obtain

$$
\begin{aligned}
\dot{\mathcal{J}}_{i}= & \int_{S} \epsilon_{i j k} x_{j}\left[-\rho u_{l}\left(u_{\mathrm{k}}+\epsilon_{k m n} \Omega_{m} x_{n}\right)\right. \\
& \left.-P_{t} \delta_{k l}+B_{l} B_{k} / 4 \pi\right] n_{l} d S \\
& +\left[\boldsymbol{\Omega} \times \int_{V} \rho \boldsymbol{r} \times(\boldsymbol{u}+\boldsymbol{\Omega} \times \boldsymbol{r}) d V\right]_{i} .
\end{aligned}
$$

The surface integral corresponds to the flux of angular momentum through the boundaries of the integration volume $V$, and the second term appears to account for the rotating frame.

We consider here only the case where the rotation axis is normal to the orbital plane. In this case the definitions of the rotational and orbital angular momentum coincide, albeit with a different integration volume $V$. In both cases, the angular momentum component of interest is $\mathcal{J}_{z}$, which is the component aligned with the rotation axis $\Omega / \Omega$. The second term of Equation (26) vanishes for $\dot{\mathcal{J}}_{z}$, leaving only the surface integral balancing the evolution of the angular momentum contained in volume $V$. If the system is in a steady state, or slowly evolving (which will be justified here a posteriori), $\dot{\mathcal{J}}_{z} \approx 0$ and as a result this surface integral is zero as well.

We now consider a volume $V$ bounded by two spherical surfaces $S_{P}$ and $S$, centered on the location of the orbiting planet, of spherical radii $R_{P}$ and $R\left(R_{P}\right.$ being typically the planetary radius). For a slowly evolving system (or a steadystate system), using Equation (26), we deduce that the torque 
applied to the planet can then be simply written as (see also Mestel \& Selley 1970; Vidotto et al. 2014)

$$
\begin{aligned}
\mathcal{T}= & \int_{S} \epsilon_{i j k} x_{j}\left[\rho u_{l}\left(u_{\mathrm{k}}+\epsilon_{k m n} \Omega_{m} x_{n}\right)\right. \\
& \left.+P_{t} \delta_{k l}-B_{l} B_{\mathrm{k}} / 4 \pi\right] n_{l} d S .
\end{aligned}
$$

This final expression can then be rewritten in any desired system of coordinates, for any surface $S$ enclosing the planet.

\section{REFERENCES}

Auclair-Desrotour, P., Le Poncin-Lafitte, C., \& Mathis, S. 2014, A\&A, 561, L7 Barker, A. J., \& Ogilvie, G. I. 2011, MNRAS, 417, 745

Baruteau, C., Crida, A., Paardekooper, S. J., et al. 2014, Protostars and Planets VI, ed. H. Beuther, R. S. Klessen, C. P. Dullemond, \& T. Henning (Tuscon, AZ: Univ. Arizona Press), 667

Bolmont, E., Raymond, S. N., Leconte, J., \& Matt, S. P. 2012, A\&A, 544, 124 Bouvier, J., \& Cébron, D. 2015, MNRAS, 453, 3720

Cohen, O., Drake, J. J., Glocer, A., et al. 2014, ApJ, 790, 57

Cohen, O., Drake, J. J., Kashyap, V. L., Sokolov, I. V., \& Gombosi, T. I. 2010, ApJL, 723, L64

Cohen, O., Ma, Y., Drake, J. J., et al. 2015, ApJ, 806, 41

Cuntz, M., Saar, S. H., \& Musielak, Z. E. 2000, ApJL, 533, L151

Damiani, C., \& Lanza, A. F. 2015, A\&A, 574, A39

Drell, S. D., Foley, H. M., \& Ruderman, M. A. 1965, JGR, 70, 3131

Evans, C. R., \& Hawley, J. F. 1988, ApJ, 332, 659

Fares, R., Donati, J.-F., Moutou, C., et al. 2010, MNRAS, 406, 409

Ferraz-Mello, S., Tadeu dos Santos, M., Folonier, H., et al. 2015, ApJ, 807, 78

Goldreich, P., \& Lynden-Bell, D. 1969, ApJ, 156, 59

Grießmeier, J. M., Zarka, P., \& Spreeuw, H. 2007, A\&A, 475, 359

Guenel, M., Mathis, S., \& Remus, F. 2014, A\&A, 566, L9

Ip, W.-H., Kopp, A., \& Hu, J.-H. 2004, ApJL, 602, L53

Jia, X., Walker, R. J., Kivelson, M. G., Khurana, K. K., \& Linker, J. A. 2008, JGR, 113, 06212

Keppens, R., \& Goedbloed, J. P. 1999, A\&A, 343, 251

Kopp, A., Schilp, S., \& Preusse, S. 2011, ApJ, 729, 116

Laine, R. O., \& Lin, D. N. C. 2011, ApJ, 745, 2

Laine, R. O., Lin, D. N. C., \& Dong, S. 2008, ApJ, 685, 521

Lanza, A. F. 2009, A\&A, 505, 339

Lanza, A. F. 2010, A\&A, 512, 77

Lanza, A. F., \& Shkolnik, E. L. 2014, MNRAS, 443, 1451

Lecavelier des Etangs, A., Sirothia, S. K., Gopal-Krishna, \& Zarka, P. 2013, A\&A, 552, 65

Leconte, J., Chabrier, G., Baraffe, I., \& Levrard, B. 2010, A\&A, 516, A64

Lesur, G., Kunz, M. W., \& Fromang, S. 2014, A\&A, 566, A56

Lovelace, R. V. E., Romanova, M. M., \& Barnard, A. W. 2008, MNRAS, 389,1233

Mathis, S., Alvan, L., \& Remus, F. 2013, in EAS Publications Ser. 62 Internal Waves and Tides in Star-Planet Systems, ed. P. Hennebelle \& C. Charbonnel (Paris: EDP Sciences), 323
Matsakos, T., Uribe, A., \& Königl, A. 2015, A\&A, 578, A6

Matt, S., \& Balick, B. 2004, ApJ, 615, 921

Matt, S., \& Pudritz, R. E. 2008, ApJ, 678, 1109

Matt, S. P., MacGregor, K. B., Pinsonneault, M. H., \& Greene, T. P. 2012, ApJL, 754, L26

Maxted, P. F. L., Serenelli, A. M., \& Southworth, J. 2015, A\&A, 577, A90

McQuillan, A., Mazeh, T., \& Aigrain, S. 2013, ApJL, 775, L11

Mestel, L., \& Selley, C. S. 1970, MNRAS, 149, 197

Mignone, A., Bodo, G., Massaglia, S., et al. 2007, ApJS, 170, 228

Miller, B. P., Gallo, E., Wright, J. T., \& Pearson, E. G. 2015, ApJ, 799, 163

Nakhaei, M., Safaei, G., \& Abbassi, S. 2014, RAA, 14, 93

Neubauer, F. M. 1980, JGR, 85, 1171

Neubauer, F. M. 1998, JGR, 103, 19843

Owen, J. E., \& Adams, F. C. 2014, MNRAS, 444, 3761

Parker, E. N. 1958, ApJ, 128, 664

Pillitteri, I., Maggio, A., Micela, G., et al. 2015, ApJ, 805, 52

Pillitteri, I., Wolk, S. J., Sciortino, S., \& Antoci, V. 2014, A\&A, 567, A128

Pont, F. 2009, MNRAS, 396, 1789

Poppenhaeger, K., \& Wolk, S. J. 2014, A\&A, 565, L1

Preusse, S., Kopp, A., Büchner, J., \& Motschmann, U. 2006, A\&A, 460, 317

Réville, V., Brun, A. S., Matt, S. P., Strugarek, A., \& Pinto, R. F. 2015a, ApJ, 798, 116

Réville, V., Brun, A. S., Strugarek, A., et al. 2015b, ApJ, 814, 99

Saur, J., Grambusch, T., Duling, S., Neubauer, F. M., \& Simon, S. 2013, A\&A, 552, 119

Shkolnik, E., Bohlender, D. A., Walker, G. A. H., \& Collier Cameron, A. 2008, ApJ, 676, 628

Strugarek, A., Brun, A. S., Matt, S. P., \& Réville, V. 2014a, in IAU Symp. 300, Nature of Prominences and their Role in Space Weather, ed. B. Schmieder, J. -M. Malherbe, \& S. T. Wu (Cambridge: Cambridge Univ. Press), 330

Strugarek, A., Brun, A. S., Matt, S. P., \& Réville, V. 2014b, ApJ, 795, 86

Strugarek, A., Brun, A. S., Matt, S. P., et al. 2014c, Proc. SFA Conf. 1411 Modelling the Corona of HD 189733 in 3D, ed. J. Ballet, F. Martins, F. Bournaud, R. Monier, \& C. Reylé, 279, 2494

Suzuki, T. K., \& Inutsuka, S.-I. 2006, JGR, 111, 06101

Trammell, G. B., Li, Z.-Y., \& Arras, P. 2014, ApJ, 788, 161

Turner, J. D., Smart, B. M., Hardegree-Ullman, K. K., et al. 2013, MNRAS, 428,678

Vidotto, A. A., Fares, R., Jardine, M., Moutou, C., \& Donati, J.-F. 2015, MNRAS, 449, 4117

Vidotto, A. A., Jardine, M., Morin, J., et al. 2014, MNRAS, 438, 1162

Vidotto, A. A., Opher, M., Jatenco-Pereira, V., \& Gombosi, T. I. 2009, ApJ, 703, 1734

Vidotto, A. A., Opher, M., Jatenco-Pereira, V., \& Gombosi, T. I. 2010, ApJ, 720,1262

Washimi, H., \& Shibata, S. 1993, MNRAS, 262, 936

Yelle, R., Lammer, H., \& Ip, W.-H. 2008, SSRv, 139, 437

Zarka, P. 2007, P\&SS, 55, 598

Zhang, M., \& Penev, K. 2014, ApJ, 787, 131 